\section{GENETICS OF CANCER}

$\mathrm{O}^{\mathrm{N}}$ $\mathrm{E}$ of the many problems arising from the study of tumour transplantation and of chemical carcinogens is the variable degree of susceptibility to cancer shown by different animal strains. This phenomenon has focused attention upon the hereditary basis of cancer, and a great wealth of experimental data has now accrued. The aim of the recent symposium on the Genetics of Cancer held in London during June 24-25, and sponsored jointly by the Genetical Society of Great Britain and by the British Empire Cancer Campaign, was to survey this field and direct attention to the significance of such recent discoveries in genetics as, for example, the role of cytoplasmic particles in heredity, differentiation and disease.

The symposium could cover only certain selected groups of problems, and the papers presented centred around three main topics: (1) the inheritance of tumours in animals, (2) the genetical aspects of virusinduced and chemically induced tumours, and (3) the inheritance of cancer in man.

(1) The genetical basis of tumour transplantation in mice was discussed by Dr. P. A. Gorer (London), according to whom tumour-transplantability depends on a number of genes, some of which determine the production of specific antigens, identified as the 'histo-compatibility' antigens. The high rate of polymorphism observed in various strains of mice by serological analysis Dr. Gorer attributed to the high rate of mutation of these genes, a fact which would also explain the frequent failures of tumour transplantation.

The genetical basis of tumour production and that of its transmission may be quite different in complexity, as was shown by Dr. G. Hogreffe (Copenhagen). Transmission of leukæmia in the 'Aka' strain of mice seems to be due to a single Mendelian factor inherited as an autosomal dominant. From transplantation experiments, however, it became apparent that the genetical basis of tumour production is much more complex.

The genetical factors involved usually operate through various intermediate agencies such as hormones. When these intermediaries are analysed in isolation the primary biological factors of tumour production may be completely obscured, and this feature was well demonstrated by Dr. R. Korteweg (Amsterdam), who dealt with the influence of oestrogenic substances on tumour susceptibility. From his data it appears that the high incidence of mammary tumours in certain strains of mice is correlated with excessive secretion of cestrogens, and that at least part of the genetically determined disposition is caused by over-production of gonadotrophic hormone.

The complexity of the hereditary basis of tumour production was further emphasized by Dr. L. Dmochowski (Leeds), who analysed the interaction of mammary tumour-inducing 'milk-factor' and genetic constitution in the development of breasttumours in certain high- and low-breast cancer strains of mice. Dr. Dmochowski arrived at the conclusion that there are two distinct sets of genetic factors, one controlling the susceptibility of breast tissue cells to the milk factor, the other controlling the propagation of the milk factor itself. The influence of hereditary factors on the production and transmission of the milk-agent was again made clear by
Dr. W. E. Heston (Bethesda, U.S.A.), whose experiments have shown that the milk-agent is not itself active, but rather prepares the substrate, that is, the mammary glands, for the initiation and mediation of tumour production by genetic factors. Dr. Heston's analysis suggests that mammary tumours may be induced in the absence of the milk-agent through the provision or construction of certain genetic and environmental patterns.

(2) The alteration of the genetic structure of cells by chemical means is a subject of relatively late development in geneties, and throws light on the possible mechanisms underlying not only 'spontaneous' or naturally occurring mutations, but chemical carcinogenesis as well. Dr. M. Demerec (Cold Spring Harbor, U.S.A.) presented evidence that when Drosophila males were exposed to aerosols of seven carcinogens (four hydrocarbons, three azo. compounds) six such compounds were found to be mutagenic, while of nine non-carcinogens (five hydro. carbons, four azo-compounds) only two were mutagens. Somewhat similar results were obtained by Dr. J. G. Carr (London), and it would seem that specific chemical compounds can certainly induce permanent alteration in the hereditary structure of cells, whether germ-cells or somatic cells. Prof. L. C. Strong (Yale University) described a multiplicity of biological effects obtained by injection of methylcholanthrene into a series of mice over many generations. Some of these effects are definite point mutations, some are non-genetic, while others are possibly induced by inhibition of embryonic morphogenesis at critical periods.

Data were also obtained by Demerec, in experiments with aerosols of carcinogens on Drosophila, indicating that there is a semi-dominant gene which determines whether or not an individual will be sensitive to the chemical induction of genetic change. There is no doubt that experimental evidence of such a diversification of gene-controlled susceptibility to chemical effects is of the greatest significance in relation to the causation of cancer. The work of Dr. A. W. Greenwood (Edinburgh) on the inheritance of susceptibility and resistance to the virus-induced Rous sarcoma showed the existence of such genetically controlled differences in fowls.

The work of Sonneborn and others has established the existence of special cytoplasmic particles which also play a part in heredity. Prof. P. B. Medawar (Birmingham), through experiments on colour transformation in guinea pigs' skin, has provided what may be another example of the particulate basis of somatic cellular heredity, and has indicated that these determining particles can behave 'infectively' towards other cells. Dr. C. D. Darlington (London) further suggested that the origin of some types of cancer can be ascribed to mutation in such cytoplasmic determ. inants. By analysing the relationship between carcinogens and viruses and its bearing on the origin of cancer, Prof. A. Haddow (London) concluded that carcinogens may induce change in a cytoplasmic particle analogous with a virus, and that the change may be brought about directly or through a primary change induced in nuclear genes. In work carried out by Prof. R. D. Passey and his collaborators at Leeds, particles of 200-350 A. in diameter were identified by the electron microscope in the milk and tumours of high-cancer strain mice, and the biological test of these particles, for tumour-inducing activity, is awaited with special interest. 
(3) In the session dealing with the inheritance of cancer in man, the symposium was presented with a general survey of the problem by Prof. T. Kemp (Copenhagen). The investigations carried out in Denmark suggest a genetical basis for predisposition to cancer, involving several genes, and furthermore that the localization, pathological features, rate of development and the time of onset of tumours may be similarly controlled. Since environmental factors also play a part in the development of particular kinds of cancer, any analysis of the heredity of cancer in man inevitably meets great difficulties. In order to compare the incidence of cancer in families with that in the total population, a critical collection of control material is required, and the difficulty of obtaining such material was stressed by several participants in the symposium. To a certain degree the problem has been solved by Prof. L. S. Penrose (London) and his collaborators, using the RegistrarGeneral's statistics for calculating the expected proportion of breast cancer and cancer deaths among relatives dying at all ages and at most periods of the immediate past : the expected death- and incidencerates for relatives can thus be compared with the observed figures. Prof. Penrose analysed the familyhistories of 510 cases of mammary cancer by this method and found that the proportion of deaths due to mammary cancer in the patients' relatives was significantly increased, the rates for other types of malignancy remaining unchanged. Similar conclusions were arrived at by Prof. D. Smithers (London) from the study of family-histories of 459 patients with cancer of the breast. Prof. Penrose concluded that a specific genetical agent is responsible for mammary cancer in man. Evidence concerning the nature of this agent was obtained from studying incidence in paternal and maternal relatives, laterality, age of onset and history of breast feeding in the patient's own infancy, from which Prof. Penrose found some indication that the specific agent might be cytoplasmic but probably not transmitted through the milk.

The studies carried out by the Danish Cancer Registry on twins, under the direction of J. Clemmesen, were presented by Dr. Th. Busk (Copenhagen). From these it seems there is a greater tendency to higher incidence of cancer among partners of identical twins afflicted with cancer than among partners of fraternal twins with cancer, and also a clear tendency for tumours in identical pairs to affect corresponding organs in both partners, whereas this is not the case among fraternal twins. Though the investigation covered about 30,000 cancer patients, only 187 twins with cancer were found suitable for analysis.

It is hoped that the investigations reported at the symposium will direct attention to the importance of recording comprehensive family histories. The need for collaboration in these matters between physicians, geneticists and statisticians was illustrated in a paper by Dr. P. C. Koller (London), who presented two family-histories of xeroderma pigmentosum, one showing an unusually mild manifestation of the disease. The distribution of this condition among the two sexes and the unusual pathological features throw further light on its genetical basis and suggest that we may need to modify Haldane's concept of partial sex-linkage in respect of this particular disease.

The proceedings of the symposium will later be published in the British Journal of Cancer.

P. C. Koller

\section{THE $R h$ FACTOR OF BLOOD}

$\mathrm{D}$ URING the summer meeting of the British Medical Association in Cambridge, the pathological section held a discussion on "Recent Advances in our Knowledge of the Rh Factor". Only the opening speaker kept strictly to this title, the other two invited speakers reading papers on closely related topics; therefore each contribution will be described separately.

Prof. D. F. Cappell, of Glasgow, first briefly described the history of the discovery of the $R h$ factor. Landsteiner and Wiener, seeking to extend knowledge of the complexities of the agglutinogen $M$ in man, immunized guinea pigs with the blood of rhesus monkeys. Surprisingly, they found that the resulting anti-rhesus serum agglutinated 85 per cent of human bloods and that the agglutinogen ' $R h$ ' so defined was unconnected with $M$. Almost immediately afterwards Wiener and Peters demonstrated the clinical importance of this antigen by showing that, in each of four cases where repeated blood transfusions had been followed by hæmolytic reactions, the cause lay in the sensitization of the patient to the $R h$ antigen. Levine and his co-workers then showed that the dreaded intra-group transfusions of women in the puerperium were also the result of iso-immunization to $R h$, and they directed attention to the almost invariable association of such reactions with death of the fotus or the occurrence of icterus gravis neonatorum. They produced striking statis. tical evidence that in these cases the $R h$ negative mother became sensitized during pregnancy to the $R h$ antigen in the blood of her foetus and formed $R h$ antibodies.

Prof. Cappell suggested that the term $R h$ factor should be reserved for the antigenic component common to man and the rhesus monkey, and the term $R$ h blood group should be used to include the whole complex of antigens which had been shown to occur together in different combinations in man. Prof. Cappell now turned to the work of Prof. R. A. Fisher and emphasized that it was he who had first recog. nized the patterm behind the complex reactions within the $R h$ groups. Fisher first noted that the reactions of different bloods with two of the four $R h$ sera then discovered were antithetical, and he postulated that the alternative reactions were due to the presence of an antigen $C$ in some bloods and of an allelomorph $c$ in others. Fisher postulated the existence of two other pairs of allelomorphic antigens, $D$ and $d$ and $E$ and $e$, which determined positive or negative reactions with the two other kinds of sera then known, which he called anti- $D$ and anti- $E$. He predicted the discovery of two further antibodies, anti- $d$ and anti-e, and the correctness of his entire conception was soon demonstrated when these predictions were fulfilled. Fisher's original scheme had had to be extended since Race's discovery of further allelomorphs $C^{w}, C^{u}$ and $c^{v}$ at the $C / c$ locus and Stratton's discovery, recently extended by Race, of further allelomorphs at the $D$ locus.

Prof. Cappell emphasized the importance of a pure anti- $D$ serum for classifying bloods as $R h$ positive or negative. For clinical purposes the important thing was whether a person was $D$ positive or negative, for all $D$ negative persons could form anti- $D$, and sensitization to $D$ was far commoner than sensitization to the other $R h$ antigens. Thus, a person of type Cde/cde might at one time have been considered $R h$ positive because of the possession of $C$; 